\title{
Pathological changes in natural infection of pheasants with highly pathogenic avian influenza A (H5N8) in Bulgaria
}

\author{
Georgi M. Stoimenov ${ }^{1}$, Gabriela V. Goujgoulova ${ }^{2}$, Branimir Nikolov ${ }^{3}$, \\ Kalin Hristov ${ }^{4}$, Atanaska Teneva ${ }^{5}$ \\ ${ }^{1}$ Department of Infectious Pathology and Food Hygiene, ${ }^{3}$ Department of Clinical Pathology, \\ ${ }^{4}$ Department of Surgery, Radiology, Obstetrics and Gynaecology, Faculty of Veterinary Medicine, \\ ${ }^{5}$ Department of Plant Protection, Faculty of Agronomy, \\ University of Forestry, 1796 Sofia, Bulgaria \\ ${ }^{2}$ National Diagnostic Research Veterinary Medical Institute, 1606 Sofia, Bulgaria \\ georgi.stoimenov.vm@gmail.com
}

Received: June 17, 2019

Accepted: November 22, 2019

\begin{abstract}
Introduction: The study of histopathological changes caused by influenza A (H5N8) viral infection in bird species is essential for the understanding of their role in the spread of this highly infectious virus. However, there are few such studies under natural conditions in minor gallinaceous species. This article describes the pathomorphological findings in Colchis pheasants infected naturally with H5N8 during an epizootic outbreak in Bulgaria. Material and Methods: Samples of internal organs of 10 carcasses were collected for histopathological and immunohistochemical evaluation, virus isolation and identification, and nucleic acid detection. Results: Consistent macroscopic findings were lesions affecting the intestine, heart, lung, and pancreas. Congestion and mononuclear infiltrate were common findings in the small intestine, as were necrosis and lymphoid clusters in the lamina propria of the caeca. Congestion with small focal necrosis and gliosis with multifocal nonpurulent encephalitis were observed in the brain. Myocardial interstitial oedema and degenerative necrobiotic processes were also detected. Immunohistological analysis confirmed systemic infection and revealed influenza virus nucleoprotein in all analysed organs. Conclusion: Variable necrosis was observed in the brain, liver, trachea, heart, small intestine, and caeca. Viral antigen was commonly found in the brain, heart, lung and trachea. Contact with migrating waterfowls was suspected as a reason for the outbreak.
\end{abstract}

Keywords: pheasants, avian influenza A virus, histopathology, immunohistochemistry, Bulgaria.

\section{Introduction}

Avian influenza, commonly known as fowl plague, is caused by viruses with segmented, negativesense, single-stranded RNA genomes belonging to the influenza virus A genus of the Orthomyxoviridae family. Avian influenza viruses (AIVs) are divided into subtypes based on the antigenic surface glycoproteins haemagglutinin (HA) and neuraminidase (NA). To date, 16 subtypes of HA (H1 to H16) and 9 of NA (N1 to N9) have been identified in birds (2). AIVs are classified as highly pathogenic for poultry when the intravenous pathogenicity index in six-week-old chickens is either greater than 1.2 or causes at least $75 \%$ mortality in four-to-eight-week-old chickens infected intravenously. The same classification applies when the characteristic motif of basic amino acids in the cleavage site of HA is identified after sequence analysis (19).

H5N8 subtype clade 2.3.4.4 was first detected in domestic poultry in China in 2010. By 2014, H5N8 highly pathogenic avian influenza (HPAI) viruses had caused a series of outbreaks among domestic ducks, chickens, geese, and wild birds in South Korea, and outbreaks followed in Japan, China, Europe, and North America $(13,15)$. 
In early 2014 in its mass distribution in South Korea, the new HPAI H5N8 virus caused disease on poultry and wildlife farms, resulting in high mortality $(10,11,12)$. The earlier HPAI H5N8 viruses of clade 2.3.4.4 clustered into two groups: the Buan-like group A sharing some identity with A/broilerduck/Korea/Buan2/2014 and the Gochang-like group B sharing some identity with A/breederduck/Korea/Gochang1/2014 (14). Different genotypes were subsequently detected during the 2016 wave of HPAI H5N8 virus, which included H5N8 reassortants being reported in Russia (14), Germany (21), India (18), and Italy (5).

The history of HPAI in Bulgaria commenced in 2006 with the occurrence of H5N1 in swans and geese (6). In 2010, H5N1 was reported in common buzzards (16) and in 2015 in Dalmatian pelicans (25). However, these cases were observed in wild birds, with only one outbreak in domestic chickens occurring in a backyard farm and no ensuing mass distribution or dissemination of the disease.

The epizootic wave of HPAI H5N8 at the end of 2016 and beginning of 2017 did not spare Bulgaria. The geographical location of this country makes it an important migration spot for migratory birds crossing Europe, Asia, and Africa. HPAI virus serotype H5N8 was detected in Bulgaria for the first time on $19^{\text {th }}$ of December 2016 in the Vidin region. By the time the wave abated, many epizootic outbreaks in domestic and wild birds had been confirmed in 15 different administrative regions of Bulgaria. Large farms with fattening ducks used for foie gras production were mainly affected. Thousands of birds were diagnosed and subsequently destroyed as mandated by the programme for eradication and control of HPAI. A wildlife farm cultivating Colchis pheasants (Phasianus colchicus) for hunting in the village of Trunkovo in the Yambol district was also affected, and as such serves as the object of our study.

HPAI viruses are known to cause a variety of septicaemic and necroinflammatory changes affecting the visceral organs and skin in gallinaceous species. In these species, it is associated with high morbidity and mortality (28). It has been established that wild waterfowl play a key role in the ecology of the disease, as they are the main reservoir and vector of the infection $(8,26)$.

The study of histopathological changes caused by H5N8 viral infection in various bird species is essential for the understanding of their role in the spread of this highly infectious virus. In particular, research on the game birds reintroduced into different regions of the country would help in understanding their role in HPAI epidemiology.

The body of pathology studies in minor gallinaceous species such as pheasants is very limited (2), especially under natural conditions (1). Understanding the pathogenesis, transmission, and intra-host evolutionary dynamics of new HPAI viruses in different avian species (including game birds) is paramount. The goal of this report is to document gross and histological lesions and antigen distribution in Colchis pheasants that died during recent outbreaks of HPAI in Bulgaria. Game bird farms represent a very small commercial sector in Bulgaria, but are a sector that also suffers from HPAI epizootics. The identification of this sector's biological potential as an epidemiological link between the waterfowl reservoir and the commercial chicken and mule duck populations is anticipated, with the ultimate goal of refining surveillance in these populations to enhance early detection, management, and control in future HPAI outbreaks.

\section{Material and Methods}

A total of 30 carcasses of birds showing clinical signs were investigated pathologically. Samples of internal organs of 10 carcasses were collected for histopathological evaluation. Samples included the heart, trachea, lung, liver, pancreas, small intestine, caecum, and brain. The carcasses, all in good condition, were necropsied, and their visceral organs and brains were examined macroscopically for gross lesions. Tissues in good post-mortem condition were collected aseptically. Half of each selected organ was used for virus isolation (VI) and PCR, and half for histopathology and immunohistochemistry. For VI, a $10 \%$ suspension $(\mathrm{w} / \mathrm{v})$ of ground sample was prepared in minimum essential media ( $\mathrm{pH} 7.2-7.4)$ supplemented with streptomycin $(200 \mathrm{mg} / \mathrm{L})$, penicillin $\mathrm{G}(2 \times 106 \mathrm{IU} / \mathrm{L})$, nystatin dehydrate $(0.5 \times 106 \mathrm{IU} / \mathrm{L})$, polymyxin $\mathrm{B}$ $\left(2 \times 10^{6} \mathrm{IU} / \mathrm{L}\right)$, gentamicin sulphate $(250 \mathrm{mg} / \mathrm{L})$, and sulphamethoxazole $(200 \mathrm{mg} / \mathrm{L})$. Organs from each carcass were microbiologically tested to exclude bacteriological infection as a cause of mass mortality in these bird species.

Virus isolation and identification. After homogenisation, the samples were centrifuged at $800 \mathrm{~g}$ for $10 \mathrm{~min}$ at $4{ }^{\circ} \mathrm{C}$. This was followed by inoculation of $200 \mu \mathrm{L}$ of the supernatant from each organ sample into the allantoic cavity of three 10-day-old embryonated chicken eggs. The infected embryos were incubated at $36^{\circ} \mathrm{C}$ for up to 96 hours and checked daily. All chicken embryos were found to be dead after 24-48 hours. Their allantoic fluids were tested for haemagglutination activity via a haemagglutination assay (HA), and the HA-positive allantoic fluids were examined for haemagglutination inhibition (HI) in an assay using four haemagglutination units per well and hyperimmune standard serum $(\mathrm{H} 5 \mathrm{~N} 1, \mathrm{H} 5 \mathrm{~N} 3)$ produced by Instituto Zooprofilattico delle Venezie $(4,17)$. The standard OIE procedure was followed for both the HA and $\mathrm{HI}$ assays (19).

Nucleic acid detection. The supernatants of the tissue homogenates were tested using real time reverse transcription PCR (rRT-PCR) for detection of the AIV 
matrix gene. RNA extractions were performed with the High Pure FFPET RNA Isolation Kit (Roche Diagnostics, Mannheim, Germany). We performed rRT-PCR with the AcuFlock Influenza A Virus realtime RT-PCR kit for M - gene (using the specific primers and probe included in the kit) following the manufacturer's protocol. Virus subtyping for H5 (24) and N8 (9) was performed with a Qiagen One-Step RTPCR Kit (Qiagen, Hilden, Germany), with samples positive for the $\mathrm{M}$ - gene according to the Animal and Plant Health Agency, UK protocol.

Histopathology. Tissue samples were immediately fixed in $10 \%$ buffered formalin, routinely dehydrated, paraffin embedded, sectioned at $5 \mu \mathrm{m}$, and stained with haematoxylin and eosin (H\&E). Duplicate sections were immunohistochemically analysed to determine the distribution of influenza virus antigens in individual tissues. Briefly, sections were stained with a mouse monoclonal antibody against influenza A virus nucleoprotein (AA5H anti-influenza A virus nucleoprotein antibody, Abcam, Cambridge, MA, USA), followed by a biotinylated goat anti-mouse IgG secondary antibody. Bound antibodies were detected with an avidin-biotin detection system (Ventana Medical Systems, Oro Valley, AZ, USA). The RedMap kit (Ventana Medical Systems) served as the substrate chromogen. Histopathological lesions were observed and documented with a DM 2500 microscope (Leica Microsystems, Wetzlar, Germany) equipped with a digital camera and original software.

\section{Results}

There were 5,637 adult pheasants on the affected Colchis pheasant farm at the time of infection. Of these, 500 birds (approximately 9\%) showed nervous signs of infection (ataxia, opisthotonus) and watery diarrhoea, and died suddenly. The disease was fatal in all of the affected birds. The remaining 5,137 pheasants were humanely destroyed.

Virus isolation and identification. H5N8 virus was isolated from the visceral organs and brain of affected pheasants. In all cases of isolated H5N8, the virus killed chicken embryos within $24-48 \mathrm{~h}$ following allantoic cavity inoculation in the first passage. Detailed inspection showed petechial haemorrhages throughout the body and developmental delay. The allantoic fluid from dead embryos was tested for haemagglutination activity, and after $\mathrm{HI}$ assays with different positive sera, it was found that this isolate was of the H5 subtype.

Nucleic acid detection. PCR for detection of M-gene following by specific rRT-PCR for H5N8analysis showed that the H5N8 AIV was present in the visceral organs of all birds examined in this study.

Gross lesions. The macroscopic changes observed in necropsies of pheasants naturally infected with HPAI H5N8 virus showed varying severity. A common finding in all birds examined were lesions affecting the heart. Well-defined white and discoloured areas of varying sizes were found in the myocardium. Frequently, petechial haemorrhages were found on the epicardial surface of the base of the heart, as well as varying amounts of yellowish fluid in the pericardial sac. The liver had well-expressed hyperaemia and singular focal necrosis (Fig. 1). Lung inspection revealed congestion complicated by swelling and singular haemorrhages (Fig. 1). The spleen was congested and enlarged. The small intestine had a diffusely hyperaemic mucosa and haemorrhagic contents mixed with a large amount of mucus. Diffuse subserosal haemorrhages in the caeca were also observed (Fig. 2). The surface of the pancreas was streaked with red-brown spots of varying sizes and singular petechial haemorrhages. The tracheal mucosa was oedematous and hyperaemic with a large amount of bloody viscous secretion.

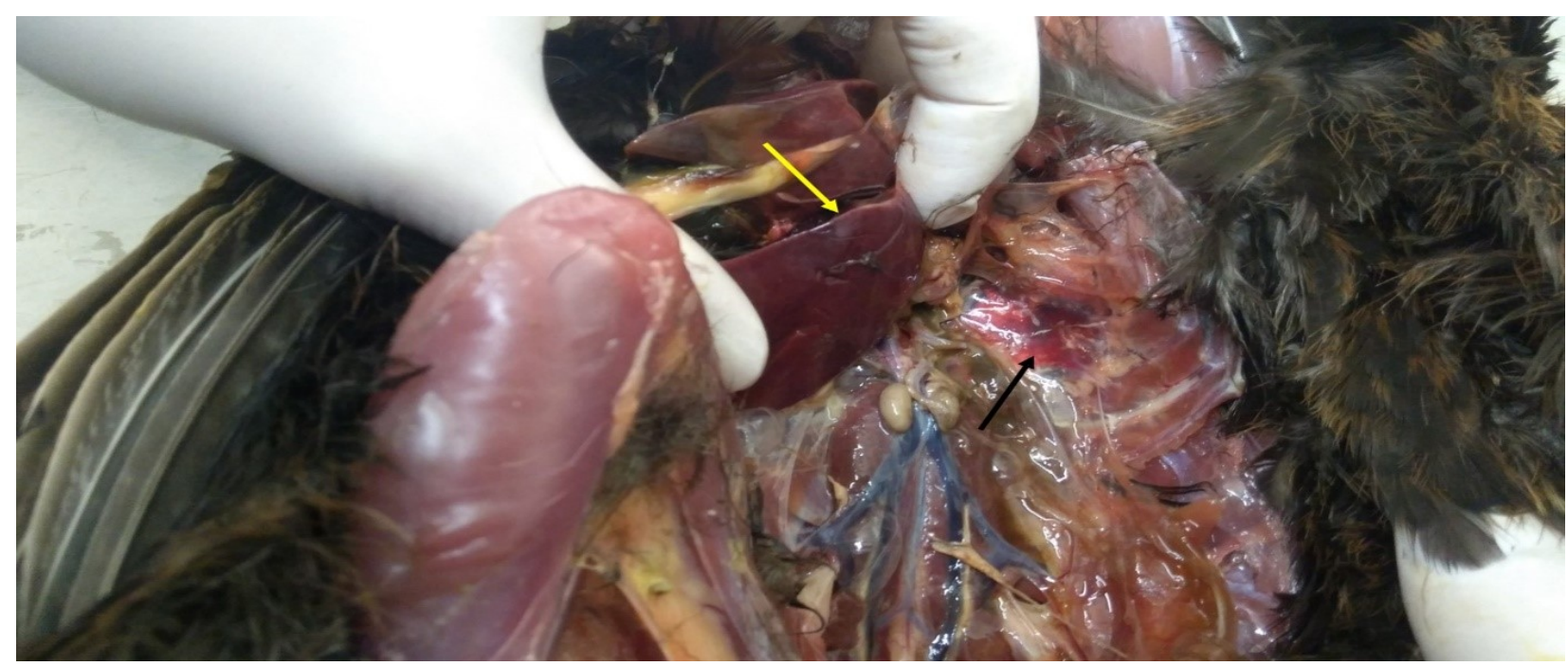

Fig. 1. Congestion, complicated by haemorrhage in the lung (black arrow) and focal hepatic necrosis (yellow arrow) 


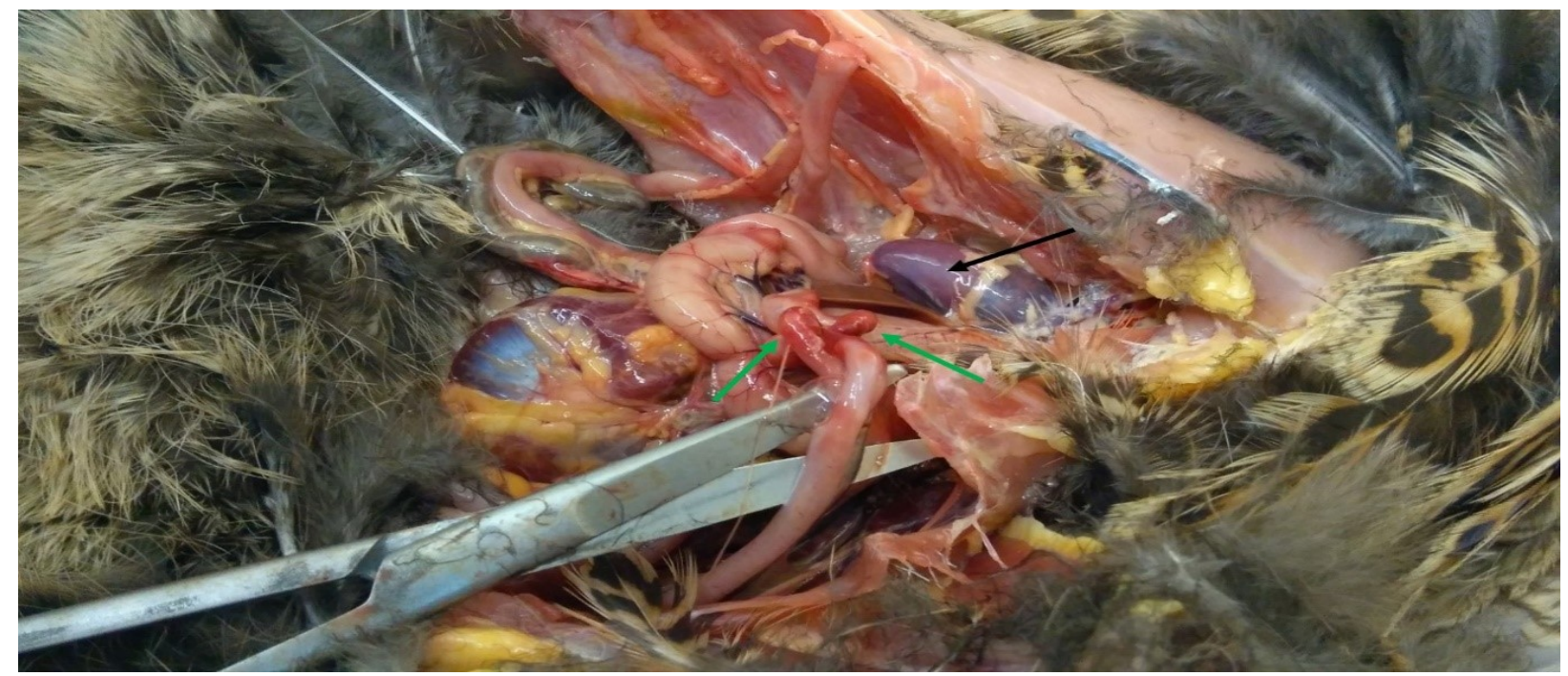

Fig. 2. Diffuse subserosal haemorrhages in the caeca (green arrows) and dystrophic and necrotic changes in the heart (black arrow)

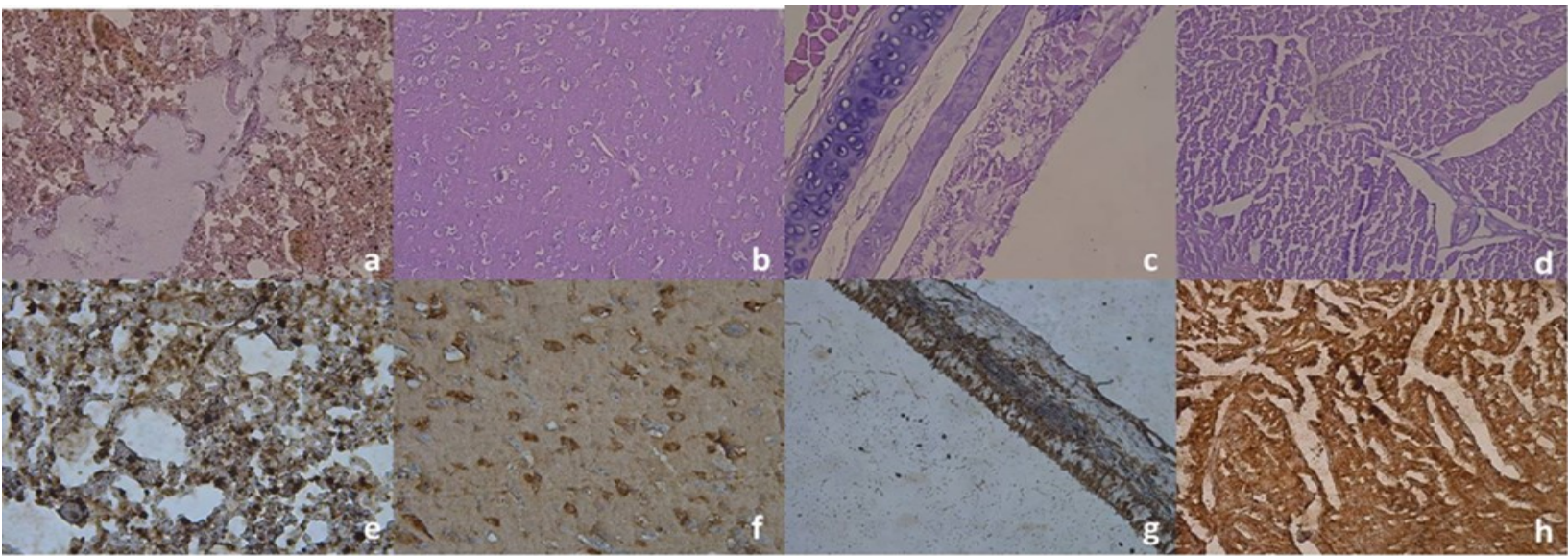

Fig. 3. Histopathological and IHC changes: a) lung - haemorrhagic pneumonia, oedema, emphysema, and atelectasis, $100 \times$; b) brain perivascular and pericellular oedema, $200 \times$; c) trachea - acute tracheitis, with epithelial desquamation, $200 \times$; d) myocardium - interstitial oedema, $100 \times$; e) lung - IHC expression of anti-influenza A virus nucleoprotein antibody, $200 \times$; $\mathrm{f}$ ) brain - IHC expression of anti-influenza A virus nucleoprotein antibody, $200 \times ; \mathrm{g}$ ) trachea $-\mathrm{IHC}$ expression of anti-influenza A virus nucleoprotein antibody, $100 \times$; h) myocardium - IHC expression of anti-influenza A virus nucleoprotein antibody, $200 \times$

Histopathological findings. The lung was characterised by well-expressed congestion and pulmonary oedema (Fig. 3a). The brain was congested with small focal necrosis and gliosis with multifocal non purulent encephalitis (Fig. 3b). Hyperaemia with oedema and loss of mucoid glands, combined with lymphocytic-plasmatic proliferate were observed regularly in the trachea (Fig. 3c). Myocardial interstitial oedema and degenerative necrobiotic processes were detected (Fig. 3d). Dystrophic and necrotic changes in hepatocytes were observed in the liver. Congestion and mononuclear infiltrates were a common finding in the small intestine, as well as necrosis of the lymphoid clusters in lamina propria. Immunohistological analysis confirmed systemic infection and revealed influenza virus nucleocapsid protein in all analysed organs: the lung 10/10 (Fig. 3a), brain 10/10 (Fig. 3b), tracheal epithelia 10/10 (Fig. 3c), and cardiomyocytes 10/10
(Fig. 3d). Immunoreactivity was observed in areas with or without microscopic lesions.

\section{Discussion}

The circumstances combined to indicate a strong threat of outbreaks of HPAI A H5N8 in Bulgaria, and the disease duly broke out in 2016. Cases of infection were reported both in wild migratory birds and in poultry in commercial and backyard farms. Given the complicated epidemic situation in Asia and Europe, and the passage through Bulgaria of two major migratory routes from Asia to Africa, this was an expected scenario. The introduction of the infectious agent onto duck farms and other industrial poultry farms was most likely due to wild migratory birds. At the beginning of 2017, HPAI A H5N8 was found in pheasants on 
a game farm in the Yambol region. The proximity of the farm to the Trankovo Dam, where many wild waterfowl spend the winter, was very instrumental in the introduction of the infection onto the farm. In addition, detection of the virus in populations of wild migratory birds during 2016 and 2017 across Europe and Bulgaria strongly supports this hypothesis.

A recurrent histopathological finding in our study was the lesions affecting the heart. In addition to petechial haemorrhages on the epicardial surface of the basal part of the heart, which is often found by other authors (7) in swans and other wild waterfowl (12), our research shows interstitial myocardial oedema and dystrophic and necrotic changes in cardiomyocytes. Myocardial damage and the severity of changes in it are associated with impaired epithelium and increased blood vessel permeability. Similar changes have been observed in chickens as a result of systemic infection with HPAI. Regarding the nature of the established pathomorphological findings, the most dominant in incidence and intensity in our cases were myocardial lesions. In the pathomorphological aspect, they differ significantly from those described as a result of HPAI A (H5N8) infection in swans and other wild waterfowl (12). In those cases, the lesion manifestations were expressed mainly in subepicardial haemorrhage and myocarditis, whereas in all the materials we studied, besides haemorrhages, the predominant lesion was the established intermyofibrillar oedema, which was even noticeable macroscopically. Severe manifestations of oedema in the myocardium may be associated with endothelial damage and increased permeability of coronary vessels in this case. This is a result of a fatal systemic disease known to be caused by HPAI H5 or H7 viruses in gallinaceous birds (27). Intensive oedematous lesions could be a direct cause of atrophic and destructive myofibrillar alterations. Significant extravasation and large fluid accumulation in the pericardial sac must also evoke the possibility that the coronary endothelium is a target for HPAI viruses. IHC examinations validate the myocardial lesions as infarction and find multifocal necrosis here to be primarily associated with virus replication and not with vascular changes.

The lungs showed evidence of marked congestion, oedema, and haemorrhage. Congestion and pulmonary oedema are non-specific changes most likely related to heart failure due to necrotising myocarditis. Demonstration of virus antigen in the pulmonary endothelium by IHC allows us to conclude that these lesions are specific to virus infection. Highly pathogenic avian influenza viruses can cause damage in cells and lead to cell death through necrosis or apoptosis. Necrosis is associated with direct virus replication in cells due to high accumulation of viral nucleoprotein in the cytoplasm and nucleus of infected cells (27).

Changes in the liver characteristic of dystrophic and necrobiotic changes fully correspond to those observed with HPAI H5N8 infection found in wild waterfowl (12).

The clinical neurological signs and observed congestion with small areas of necrosis and gliosis with multifocal encephalitis confirm the neurotropism of the virus. Such results were also found in other studies in various bird species as a consequence of HPAI H5N8 infection (12) and more pertinently were found in minor gallinaceous species (1). The number of pheasants with encephalitis, in association with high levels of virus as detected by immunohistochemistry, suggests that the virus is highly neurotropic, as also shown by previous studies $(3,20)$. The mechanisms of dissemination of the virus in the brain are not yet fully understood. However, the explanation that the route of infection also affects the route of dissemination is plausible. Experimental studies on mice, ferrets, and chickens suggest that influenza viruses can enter the central nervous system haematogenously, via peripheral nerves (23) and the olfactory route (22). These observations can serve as the foundation for the hypothesis that the main route of AIV infection of the brain in pheasants is through the circulatory system.

In conclusion, this study documents the clinicopathological findings and viral antigen distribution of natural HPAI H5N8 infection in pheasants. All the birds exhibited multifocal nonpurulent encephalitis, myocardial interstitial oedema and degenerative necrobiotic processes, and hyperaemia with oedema and loss of mucoid glands, combined with lymphocytic-plasmatic proliferate in the trachea. Variable necrosis was observed in the brain, liver, trachea, heart, small intestine, and caeca. Viral antigen was commonly found in the brain, heart, lung, and trachea. The contact between the pheasants and migrating waterfowl was suspected as a reason for the outbreak.

Conflict of Interests Statement: The authors declare that there is no conflict of interests regarding the publication of this article.

Animal Rights Statement: None required.

Financial Disclosure Statement: This work was supported by the Coordinated Research Project (CRP) D32030: "Use of Stable Isotopes to Trace Bird Migrations and Molecular Nuclear Techniques to Investigate the Epidemiology and Ecology of the Highly Pathogenic Avian Influenza" of the International Atomic Energy Agency (IAEA), implemented through the Animal Production and Health Section of the Joint FAO/IAEA Division.

This research work was carried out with the support of the University of Forestry and project BG05M2OP001-2.009-0034 titled "Support for the development of scientific capacity in the University of Forestry" and the Operational Programme "Science and Education for Smart Growth", co-funded by the 
European Union through the European Structural and Investment Funds.

Acknowledgments: The authors would like to thank Dr John Bianco for critical reading of the manuscript.

\section{References}

1. Bertran K., Dolz R., Majó N.: Pathobiology of avian influenza virus infection in minor gallinaceous species: a review. Avian Pathol 2014, 43, 9-25.

2. Bertran K., Lee D.H., Pantin-Jackwood M.J., Spackman E., Balzli C., Suarez D.L., Swayne D.E.: Pathobiology of clade 2.3.4.4 H5Nx high-pathogenicity avian influenza virus infections in minor gallinaceous poultry supports early backyard flock introductions in the Western United States in 2014-2015. J Virol 2017, 91, 21, e00960-17. doi: 10.1128/JVI.00960-17.

3. Brown J.D., Stallknecht D.E., Beck J.R., Suarez D.L., Swayne D.E.: Susceptibility of North American ducks and gulls to H5N1 highly pathogenic avian influenza viruses. Emerg Infect Dis 2006, 12, 1663-1670.

4. Comin A., Toft N., Stegeman A., Klinkenberg D., Marangon S.: Serological diagnosis of avian influenza in poultry: is the haemagglutination inhibition test really the "gold standard"? Influenza Other Resp Viruses 2013, 7, 257-264.

5. Fusaro A., Monne I., Mulatti P., Zecchin B., Bonfanti L., Ormelli S., Milani A., Cecchettin K., Lemey P., Moreno A., Massi P., Dorotea T., Marangon S., Terregino C.: Genetic diversity of highly pathogenic avian influenza A (H5N8/H5N5) viruses in Italy, 2016-2017. Emerging Infect Dis 2017, 23, 1543-1547. doi: 10.3201/eid2309.170539.

6. Goujgoulova G., Oreshkova N.: Surveilance on avian influenza in Bulgaria. Avian dis 2007, 51, 382-386.

7. Harder T., Maurer-Stroh S., Pohlmann A., Starick E., HörethBöntgen D., Albrecht K., Pannwitz G., Teifke J.P., Gunalan V., Lee R.T.C., Sauter-Louis C., Homeier T., Staubach C., Wolf C., Strebelow G., Höper D., Grund C., Conraths F.J., Mettenleiter T.C., Beer M.: Influenza A (H5N8) virus similar to strain in Korea causing highly pathogenic avian influenza in Germany. Emerging Infect Dis 2015, 21, 860-863. doi:10.3201/eid2105. 141897.

8. Hinshaw V.S., Bean W.J., Webster R.G., Sriram G.: Genetic reassortment of influenza $A$ viruses in the intestinal tract of ducks. Virology 1980, 102, 412-419.

9. Hoffmann B., Hoffmann D., Henritzi D., Beer M., Harder T.C.: Riems influenza A typing array (RITA): an RT-qPCR-based low density array for subtyping avian and mammalian influenza A viruses. Sci Reports 2016, 6, 27211. doi:10.1038/srep27211.

10. Jeong J., Kang H.M., Lee E.K., Song B.M., Kwon Y.K., Kim H.R.: Highly pathogenic avian influenza virus (H5N8) in domestic poultry and its relationship with migratory birds in South Korea during 2014. Vet Microbiol 2014, 173, 249-257.

11. Kang H.M., Lee E.K., Song B.M., Jeong J., Choi J.G., Jeong J., Moon O.K., Yoon H., Cho Y., Kang Y.M., Lee H.S., Lee Y.J.: Novel reassortant influenza A (H5N8) viruses among inoculated domestic and wild ducks, South Korea, 2014. Emerging Infect Dis 2, 2015, 298-304.

12. Kim H., Kwon Y., Jang I., Lee Y., Kang H., Lee E., Bae Y.: Pathologic changes in wild birds infected with highly pathogenic avian influenza A (H5N8) viruses, South Korea, 2014. Emerging Infect Dis 2015, 21, 775-780. https://dx.doi.org/10.3201/ eid2105.141967.

13. Lee D.H., Bahl J., Torchetti M.K., Killian M.L., Ip H.S., De Liberto T.J., Swayne D.E.: Highly pathogenic avian influenza viruses and generation of novel reassortants, United States, 2014-2015. Emerging Infect Dis 2016, 22, 1283-1285. doi: 10.3201/eid2207.160048

14. Lee D.H., Bertran K., Kwon J.H., Swayne D.E.: Evolution, global spread, and pathogenicity of highly pathogenic avian influenza H5Nx clade 2.3.4.4. J Vet Sci 2017, 18, 269-280. doi: 10.4142/jvs.2017.18.S1.269.

15. Lee Y.J., Kang H.M., Lee E.K., Song B.M., Jeong J.S., Kwon Y.K.: Novel reassortant influenza A (H5N8) viruses, South Korea, 2014. Emerging Infect Dis 2014, 20, 1087-1089.

16. Marinova-Petkova A., Georgiev G., Seiler P., Darnell D. Franks J., Krauss S., Webby R.J., Webster R.G.: Spread of influenza virus A (H5N1) clade 2.3.2.1 to Bulgaria in common buzzards. Emerging Infect Dis 2012, 18, 1596-1602. doi:10.3201/eid1810.120357.

17. Molesti E., Wright E., Terregino C., Rahman R., Cattoli G., Temperton N.J.: Multiplex evaluation of influenza neutralizing antibodies with potential applicability to in-field serological studies. J Immunol Res 2014, 457932. doi:10.1155/2014/457932.

18. Nagarajan S., Kumar M., Murugkar H.V., Tripathi S., Shukla S., Agarwal S., Dubey G., Nagi R.S., Singh V.P., Tosh C.: Novel reassortant highly pathogenic avian influenza (H5N8) virus in zoos, India. Emerging Infect Dis 2017, 23, 717-719. doi: 10.3201/eid2304.161886.

19. OIE: Terrestrial Manual, Chapter 2.3.4. http://www.oie.int/fileadmin/ Home/eng/Health_standards/tahm/2.03.04_AI.pdf.

20. Pasick J., Berhane Y., Embury-Hyatt C., Copps J., Kehler H., Handel K., Babiuk S., Hooper-McGrevy K., Li Y., Mai Le Q., Lien Phuong S.: Susceptibility of Canada geese (Branta canadensis) to highly pathogenic avian influenza virus (H5N1). Emerging infect dis 2007, 13, 1821-1827.

21. Pohlmann A., Starick E., Harder T., Grund C., Hoper D., Globig A., Staubach C., Dietze K., Strebelow G., Ulrich R.G., Schinkothe J., Teifke J.P., Conraths F.J., Mettenleiter T.C., Beer M.: Outbreaks among wild birds and domestic poultry caused by reassorted influenza A $(\mathrm{H} 5 \mathrm{~N} 8)$ clade 2.3.4.4 viruses, Germany, 2017. Emerging Infect Dis 2017, 23, 633-636. doi: 10.3201/eid2304.161949.

22. Schrauwen E.J.A., Herfst S., Leijten L.M., van Run P., Bestebroer T.M., Linster M., Bodewes R., Kreijtz J., Rimmelzwaan G.F., Osterhaus A., Fouchier R.A.M., Kuiken T., van Riel D.: The multibasic cleavage site in H5N1 virus is critical for systemic spread along the olfactory and hematogenous routes in ferrets. J Virol 2012, 86, 3975-3984.

23. Shinya K., Makino A., Hatta M., Watanabe S., Kim J.H., Hatta Y., Gao P., Ozawa M., Le Q.M., Kawaoka Y.: Subclinical brain injury caused by $\mathrm{H} 5 \mathrm{~N} 1$ influenza virus infection. J Virol 2011, 85, 5202-5207.

24. Slomka M.J., Pavlidis T., Banks J., Shell W., McNally A., Essen S., Brown I.H.: Validated H5 Eurasian real-time reverse transcriptase-polymerase chain reaction and its application in H5N1 outbreaks in 2005-2006. Avian Dis 2007, 51, 373-377.

25. Stoimenov G.M., Goujgoulova G.V., Nikolov B., Petrova R., Teneva A., Dimitrova I.: Histopathological findings in Dalmatian pelicans (Pelecanus crispus) naturally infected with avian influenza subtype A H5N1 in Bulgaria. J Hellenic Vet Med Soc 2017, 68, 369-376. http://dx.doi.org/10.12681/jhvms.15493.

26. Suss J., Schafer J., Sinnecker H., Webster R.G.: Influenza virus subtypes in aquatic birds of eastern Germany. Arch Virol 1994, 135, 101-114.

27. Swayne D.E., Halvorson D.A.: Influenza. In: Diseases of poultry. Edited by Y.M. Saif, A.M. Fadly, J.R. Glisson, L.R. McDougald, L.K. Nolan, D.E. Swayne, Blackwell, Ames, 2008, pp. 153-184.

28. Swayne D.E., Suarez D.: Highly pathogenic avian influenza. Rev Sci Tech 2000, 19, 463-482. 